\title{
Working With or Next to Each Other? Boundary Crossing in the Field of Information Visualisation
}

\author{
Gerard Smit, Yael de Haan, \& Laura Buijs \\ University of Applied Science Utrecht, the Netherlands \\ E-mail: yael.woortman-dehaan@hu.nl
}

\begin{abstract}
Due to the need to present information in a fast and attractive way, organizations are eager to use information visualisations. This study explores the collision between the different experts involved in the production of these visualisations using the model of trading zones supplemented with the learning mechanisms found in the boundary crossing literature. Results show that that there is not one single good solution to effective interdisciplinary cooperation in the field of information visualisation. Rather, all four types of cooperation that we distinguish - enforced, dominated, fractionated, and attuned - might work well, as long as they are adapted to the situation and the participants accept the constraints of the specific cooperation type they are engaged in. In any case the involved experts and initiators have to understand and incorporate approaches that enhance the cocreative, iterative nature of the production process. In surveying the different forms of collaboration we detect two major forms of trading zones: the one that encompasses the collaboration between an external client and a designer (external trading zone) and the trading zones within an organization between content producer and designer (internal trading zone). Both mechanisms of identifying each other's expertise and coordinating the different tasks in the production process seem beneficial for the production process.
\end{abstract}




\section{Keywords}

Data visualisation, infographics, information visualisation, multi-skilled journalists, trading zone, boundary crossing, computational thinking, newsroom studies, case study

\section{Introduction}

In current society people are often faced with an information overload due to the increasing number of media platforms and communication channels, the rise of open data and the complexity of modern network society. Several studies have shown that infographics and visualized information can counter this problem of information overload as visualisations can help people understand complex information (Heiser \& Tsversky, 2002; De Koning, Tabbers, Rikers \& Paas, 2010). Other studies have also shown the positive effect of content recall when using visualisations (Lankow, Ritchie \& Crooks, 2012). Many companies, including media organizations, businesses, and government organizations have therefore shown an exploding demand for visualisations in their communication towards their clients. However, visualising information that generates the intended effect is not an easy task as it demands know-how and skills from a range of disciplines, including writing, editing, researching, data analysis, programming and designing (Giardina \& Medina, 2013; Kirk, 2012; Segel \& Heer, 2010). Because a jack-of-all-trades is often a rarity, the crucial success of a visualisation lies in a fruitful collaboration between various disciplines (Beak, Liebowitz, \& Lewis, 2000; Mora, 2012; Weber \& Rall, 2012). Nevertheless, a different background and culture, insufficient knowledge of each other's expertise and no common language often obstruct a successful teamwork (see also Smit, De Haan, \& Buijs, 2014).

In many companies and media organizations the written content or texts still dominate over the visuals with the designers often taking a more supporting role, if there are any within the company at all. Design and programming tasks are often outsourced for specific projects. Coming from different disciplines and not understanding each other's language or work can lead to problems in the process of making a visualisation (Kleinsman, Valkenburg, \& Buijs, 2007; Weber \& Rall, 2012). It is this problem of interdisciplinary working and the collaboration between different disciplines that we want to tap into. In this research we want to understand the production process of information visualisations and focus on the problems of collaboration between the different experts involved. Through a multi-case study approach we make an in-depth analysis of the production process of visualisations and challenges a multi-disciplinary collaboration demands. Our starting point is a theoretical framework of cross-disciplinary collaboration with a focus on the concepts of trading zones and boundary 
crossing, which we will briefly discuss below, after which we present our method and results.

\section{Theoretical framework}

\section{Trading zones}

To analyse the cooperation between different disciplines we use the concept of the trading zone. It was originally an anthropological concept to describe how different cultures are able to exchange goods despite differences in language and culture. Peter Galison (1997) introduced the term to the social studies of science to analyse the communication between scientists whose paradigms are incommensurable. According to Galison two groups can agree on rules of exchange even if they ascribe utterly different significance to the objects being exchanged.

Collins, Evans, and Gorman (2007) use the metaphor of the trading zone in a more general sense to explain how communication is managed where there is a degree of incommensurability between professionals in general. The trading zone model developed by Collins et al. (2007) has two dimensions and depicts the collaboration between different experts involved. One is the extent to which power is used to enforce trade - this is the collaboration - coercion axis. The other dimension is the extent to which trade leads to a homogenous new culture with shared values - this is the homogeneity-heterogeneity axis. In this model trading zones are dynamic entities and describe the different states in which a trading zone might exist over time. Collins et al. use this model to describe possible trajectories of groups coming together around the idea of producing a common good. We adapted this model slightly, using more suitable terms for the field of information visualisations (Figure 1).

The four trading zones correspond to the quadrants formed by coercioncollaboration axe and the heterogenic-homogeny axe. The first quadrant or type of collaboration is the enforced trading zone (lower left quadrant in figure). In this type of trading zone collaboration is compulsory with a low level of homogeneity. The second quadrant or trading zone is not compulsory, but based on some level or will of two parties to collaborate, however from their own expertise and less from a joint objective. This is the so-called fractionated trading zone (upper left quadrant). In the third type of trading zone, the so-called attuned trading zone, (upper right quadrant) the experts of different disciplines are not driven by their own expertise but by the challenge posed by the object they want to produce. Collins et al. derive this from the idea that once the collaboration between two heterogenic groups goes smoothly, there is an integration of disciplines and languages, possibly leading into a new discipline. For example, 


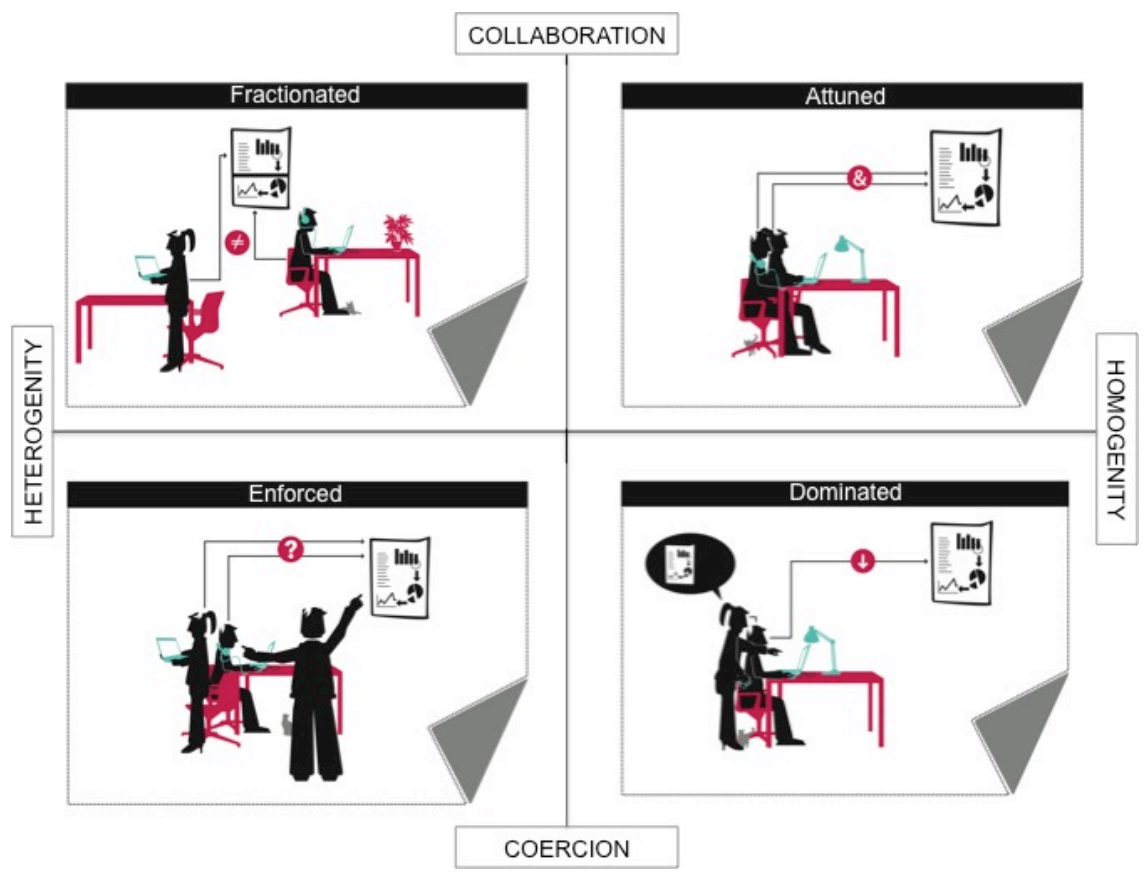

Figure 1: The four trading zones.

the field of biochemistry is an integration of biology and chemistry that have teamed up with joint objectives, which has led to the integration of two disciplines. The fourth trading zone is a combination of the coercion and homogeneity axis. We call it the dominated trading zone (lower right quadrant) ('subversive' in the Collins model). In this form of collaboration one discipline, culture or language is taken over by the other more dominant one. Or as Collins states: "Subversive trading zones operate by imposing one culture on another" (2007, p. 660).

\section{Boundary objects and interactional expertise}

Collins et al. (2007) analyse the dynamics of trading zones - that is to say the way collaboration changes over time - by adding the concepts of 'boundary object' and 'interactional expertise'. A boundary object serves as a common point of reference that is interpreted differently by the involved actors. These are objects that "both inhabit several intersecting worlds and satisfy the informational requirements of each of them" (Star \& Griesemer, 1989, p. 393). When the shared meaning of a boundary object is expanded, the actors can move into other trading patterns. Interactional expertise involves internalization of the tacit components of a strange language (Collins et al, 2007, p. 661). It is the expertise to be able to 
interact with other disciplines without being able to practice that certain discipline (Collins, 2004). Boundary objects and interactional expertise are respectively perceived as material and linguistic devices to represent the cultural baggage of the trading partner.

\section{Boundary crossing and learning mechanisms}

The overall dynamics of the trading zones could well be perceived as a form of boundary crossing to describe the way trading partners find common ground for their negotiations or move from on to another trading zone. In their literature review on boundary crossing and boundary objects Akkerman and Bakker (2011) argue that learning from each other - a perfect and simple way to describe what boundary crossing is about - involves dialogue between multiple perspectives and parties without implying or seeking homogeneity. They discern four dialogical learning mechanisms of boundaries: (a) identification, which is about understanding and learning more about other practices and expertise and how they are related to one another; (b) coordination, which is about creating cooperative and routinized exchanges between practices; (c) reflection, which is about expanding one's perspectives on the practices; and (d) transformation, which is about collaboration and co-development of (new) practices.

In our study we use the concepts of trading zones and boundary crossing to analyse how professionals in the production of visualisations exchange expertise in pursuit of a common task.

\section{Method}

To understand how different disciplines collaborate when making visualisations, we use a multi-method case study approach. Firstly, however, we conducted seven expert interviews with prominent information visualisation designers in the Netherlands who are involved in the whole process of information design to get a broad understanding of how information visualisation experts see their role and with which type of challenges they are coping with. While visualizations are not only produced by designers, we chose them as a starting point as designers are often expected to be a jack-of-all trades and be multi-skilled. Subsequently, we analysed the production process of four visualisations and also spoke to other actors involved in the process.

The first production we analysed was a visualisation made by an information visualisation agency for a foster childcare organization. In the Netherlands, in 2015 the responsibility for foster childcare will be decentralized from the national government to municipalities. The foster childcare organization wanted to visualize the way they work to inform municipality officers. The visualisation 
agency was founded by two journalists and now consists of ten content providers, people who primarily are responsible for clarifying the message and the content of the visualisation, and two designers. The second production was an interactive animation made by an animation agency for a small recycling company with the objective of informing their clients about their sustainable working process. The animation agency works with eight designers, one project manager and two founders, who also have a design background. The third case was based at a Dutch research institute for environmental policy. With approximately 250 employees (of which five are designers responsible for the information visualisations) the main task of the institute is to research environmental and spatial planning issues. We followed the production process of the visualisation of one of their annual reports. The last case we conducted was at a media broadcasting organization that produces visualisations to accompany the news items on a daily basis. In total 120 people work at the organization, of which most are journalists. The graphics departments consists of eight people who produce visuals for the news bulletins and the website.

All case studies were conducted in a similar manner with three different methods: document analysis, observations and interviews. First, written documents such as briefings, procedures, e-mails, and manuals were analysed to understand the context of the organization and the project. Second, the researchers attended numerous meetings throughout the production process and had informal talks with different people at the newsroom. Finally, semi-structured interviews were held with the people involved in the visualisation process including clients, journalists, project managers and information designers. The data collection was done in the period between January and June 2013.

\section{Results}

We will first describe the results per case. Here we elaborate on the trading between parties, what boundary objects were used, and why the trading worked or failed. This will help readers to better understand the theoretical concepts at work in our cases. We then compare the cases and make general conclusions on the production process of visualisations and the problems of interdisciplinary collaboration.

\section{Mapping the field of foster childcare by a commercial design agency}

The visualising company that was asked to visualize the way foster childcare works in the Netherlands is a rather small and informal group of people with a design and journalistic background. Their overall aim is to assist clients in clarifying complex issues and to help them visualize this. They mainly work with 
a fixed format of visualisations and information maps. The starting point of making a visualisation begins with a briefing from the client. Foster childcare was clear in that it wanted to make a decision tree to explain to municipality officials how they work, and to them the form was straightforward as they leaned on previous work of the visualization company.

Following, the content providers of the visualization agency organized two brainstorm sessions with the client and other relevant stakeholders, including a representative of the target group, in this case a municipality officer. These first two steps in the production process show that quite some time is dedicated to coming to consensus on the objective of the visualisation and the message that has to be put across. These brainstorming sessions could be seen as a way for the client and the visualization agency to understand and identify with each other (learning mechanism identification). However, it is exactly at this point where we saw that collaboration between the client and the content providers did not go too smoothly and that both parties did not really learn from each other. During the brainstorming sessions there was quite some initial disagreement on the objective of the visualisation and the trading between the parties did not really take off. The content providers took a submissive role by listening to the client and following up their wishes of making a decision tree. They focused on fine-tuning the message during the brainstorm sessions and they did not openly question whether a decision tree would be the most suitable visual. Only after two brainstorming sessions of each three hours with approximately 15 stakeholders and a first sketch of the visualisation being presented, it became clear that the decision tree might not be the most appropriate visualisation for the message that childcare wanted to communicate. The explanation could be that brainstorming does not work optimally when the participants stay captivated in their own mindset. This implies that in order for a brainstorming session to be effective and to create a boundary crossing, some form of identification is needed.

While the first part of the production process is an external collaboration between client and content providers, in the second part the content providers collaborate with designers to transform the content into a visual. The designers are not involved in the communication with the client. The content providers are the central figures in the process, communicating both to the client and to the designers. Our analysis shows that in this internal collaboration the content providers dominated over the designers (dominated trading zone). The designers clearly took a submissive role and completed the tasks that the content providers asked them to do. In this relationship there is a clear hierarchical difference where the designers take a more executive role and are less involved in the creative and analytical phase. The content providers formulated and actually made the first sketch of the visual after which the designers were responsible for the execution. What made it more difficult is that for the content providers this hierarchy is more or less evident as it fits the organizational structure and process, while the designers clearly stated that this way their work and competences are 
undermined. One designer said: 'It's a pity we weren't involved in the strategy phase of the visualisation. Now I feel more of an Indesign expert, who merely knows how to use specific software, and not so much an infographic expert who has specific design and analytical skills." In this relationship the first sketches can be seen as an initial process of identification between the content processors and the designers. Even though this identification process helps facilitate the production, it didn't improve the exchange relationship as it was only halfheartedly fulfilled. The content providers believed their sketches were efficient and effective tools in the process, but the designers would have liked to give more creative input. The difference between what the designers actually do and their perception of what their job should be, creates quite some frustration and tension, which doesn't do the overall collaboration much good.

This case shows that much time is taken to extract the objective of the visualisation and the message that has to be communicated. This delayed the process with a few months. The collaboration can mostly be typified by a dominated trading zone, with the designers fulfilling a supportive role. Nevertheless, in a small organization in which many visuals are somewhat standardized, the content providers believe the hierarchal difference and submissive role of the designer is the most efficient division of labour. This dominated trading zone can be efficient when working processes are standardised. However, both disciplines need to agree on this and identify with each other. The fact that identification did not take place obstructed or delayed a fruitful collaboration process.

Demonstrating the production process of a recycling company by a commercial animation agency

The second case we followed was also at a small design agency. However, this one focuses on animations and largely consists of employees with a design background. The project manager is responsible for the overall process and planning and the communication between client and designers. The case we followed was of a small recycling company that wanted to inform their clients about their sustainable production process. The account managers of the company used to spend a lot of time talking with prospects about the way they work and all the recycling possibilities they offer before doing business. The animation was meant to be sent to the prospects prior to the first appointment. The objective of the visualisation and the target group it was aimed at was quite clear-cut.

The production process always starts with a briefing with the client, in which the animation agency uses a standardized form to help clients outline their main goal, message, target group etc. In this case the commercial director and head of marketing communication briefed the project manager of the animation agency after which she made a first script for the story. The project manager takes a 
central role in transforming the message of the client to the designers to be able to make the most suitable animation. Once the client approves the script, animation designers get involved and storyboards are made, followed by the animations. Throughout the process, the client is informed of the concepts and is able to give feedback.

Just as with the previous case, this case consists of two collaboration forms: an external collaboration between client and project manager, and an internal collaboration between project manager and animation designer. If we look at the external collaboration it is clear that the client takes a submissive role towards the animation agency as the former believes the latter is the expert in visualising the message (dominated trading zone). The standardized form that is used to specify the goal and message of the animation is used as a practice of boundary crossing, which helped the client and animation agency identify with each other and coordinate the working process. In this case, when both parties are on the same page and the expectancies are clear, a dominated trading zone works well.

This successful border crossing and collaboration were facilitated by two factors: the size of the organizations and the roles of the parties involved. Working with a small agency in which the client also represents a small company makes the working process smoother as the involved actors can communicate quickly and informally. The roles and expectations between the involved actors were clear with a cooperative mind-set, which contributed to a fruitful collaboration.

Internally, we also see collaborative relationship between the project manager and the designer. Both parties have clearly distinguished roles, but at the same time a joint objective to create the most suitable animation. Both acknowledged each other's expertise as well. The animation designer for instance regularly asked the project manager for advice. Many (design) decisions he made were also in accordance with the project manager, since she was the one most familiar with the wishes of the client. The project manager did not claim to be the creative expert, and in communication with the client she regularly drew attention to the creative input of the animation designer.

The smooth collaboration, both internally and externally, led to the relative short production time of six weeks, which was according the initial planning.

\section{Visualising an annual report of a government organization}

The first two visualisations were done by clients who outsourced the visualisation to a design agency. This third case was conducted at a government environmental research agency that has an internal design department. Their objective was to visualize their annual report and make it more attractive. In particular, the management of the agency felt a strong need to produce more visualisations to hopefully increase the impact on government policy, and also to reach a broader 
public. For this project a number of people were involved including a group of researchers or content providers and a group of designers. In addition, they decided to ask a renowned infographic maker to assist the design process. As well, a researcher who regularly worked with the design unit was appointed to be the so-called 'transformer' with the task of coordinating the whole production process of information visualisations and to make sure the researchers and designers linked their work to each other. Previous visualisation projects had a rather linear character as the researchers first elaborated on the content after which the designers visualized the provided content. With the appointment of an overall project manager, whose task was to supervise and coordinate an increasing number of visuals, the management decided to experiment with a more cyclical process in which researchers and designers worked on the project simultaneously.

In this case, we see a first form of a rather enforced collaboration (enforced trading zone), as the management demanded that the employees work together on making the annual report more visually attractive. However, the way this cooperation should be organized or the way the visualisation process should be structured was left to the researchers and designers themselves.

For this project, the idea was that both parties work simultaneously: the researchers provide the content and write the report and the designers work on visualising the content. Throughout the process the designers and researchers came together for working sessions to adapt the content to the visualisation. These working sessions can be seen as the trading object where both parties can come to consensus and to create some form of boundary crossing. However, in practice this process did not go smoothly. This mainly had to do with the fact that the two teams, the researchers and the designers, did not have a joint objective in mind and did not feel the need to identify with the other party or experts. Both parties worked separately on their own expertise and quite independently of each other, not taking the end product and final objective into account. Also it was difficult to accommodate to each other's work, as the researchers continuously made adjustments in the report, which meant that the designers often also had to adjust the visual.

This process can be typified as a fractionated collaboration or trading zone. In this collaboration, the trading object was not sufficient for both parties to be on the same page and to be able to cross boundaries. Therefore, the role of the transformer is crucial in this collaboration as this person can mobilize people to collaborate and to find a common ground and language - in short, to provide an efficient 'trading place'. However, the fact that this person felt more part of an affinity with the infographic department, and thus was not really independent made it quite problematic to actually achieve a fruitful debate or good relationship in the team. The renowned infographic maker that was asked to assist in the creative process could also have contributed in the collaboration process as he speaks the same language as the designers. Nevertheless, this case shows that 
not only identification but also coordinating each other's task is crucial to be able to work together efficiently.

Underlying this fractionated relationship, we observed that the researchers dominated the designers with the latter clearly taking a more submissive role (dominated trading zone). This was especially visible during the joint working sessions, where the designers mostly listened to the comments the researchers had on their design concepts. When certain research concepts were unclear to the designers they often felt uncomfortable asking for further explanation. There was not only detachment in terms of expertise, but also literally there was a division in terms of physical distance. Because most of the researchers were stationed at a different research location in the Netherlands than the designers, the joint working sessions took place through videoconferencing, which to the designers did not always go smoothly. A designer said: "If I would send an email to one of the researchers for additional data, they often did not reply. They acted somewhat arrogant. Of course I have myself to blame as well, but the researchers are difficult to work with." This hierarchical difference can be explained by the fact that the core business of the agency is to conduct research. The design department is clearly a staff department. Moreover, the fact that the management accentuated the need for more visualisations, does not mean that this is also felt among the researchers. Finally, the fact that an external renowned designer was asked to assist in the design process might make the designers uncomfortable and insecure and make their own work seem less valuable.

The case shows three types of trading zones: enforced, fractionated and dominated. The fractionated offered perspectives for fruitful trading exchanges through working sessions and a transformer functioning as a trading object. However, the dominated trading zone prevailed, with the designers being overruled by the content providers and learning mechanisms were not used effectively. The result of this project is that the annual report was published with merely three visuals instead of the aimed minimum of five during a period of six months.

\section{Visuals in 24-hours broadcasting newsroom}

During the last case, we did not follow the production process of one specific visualisation, but we looked at more short-term collaborations between journalists and designers at a leading Dutch broadcasting organization as a 24-hours newsroom demands more visuals with a very short lead time. The newsroom has 120 employees. A separate graphics department of eight designers was set up approximately six years ago with the task of accompanying the news items with visuals on television and on the website. While the newsroom is an open office with the different editorial units merely separated by tables, the design unit is physically divided and situated in another room. 
Analysing the production process of several visuals, we initially observed a fractionated collaboration between infographic designer and journalist. The journalist is the one that is responsible for the news story and the content and also initiates the idea of adding a visualisation to the news item. Following, the designers are briefed and asked to make the visual. There is a clear division in tasks and the working process has a strong linear character. However, we often saw that the collaboration did not go well as both parties often had different ideas on the objective of the infographic or visual. The intention of the journalist was often to make the content more attractive by using a visual, while the infographic designer is more focused on the information function. As a designer said: "Our goal is to help journalists make their story accessible for a large public and not so that the journalist can show off with a news item that is visually attractive."

This fractionated relationship is not only obstructed due to a lack of a joint objective, but maybe more so because we saw an underlying hierarchal relationship, in which the designers clearly had a supportive and executive role. A designer illustrated this hierarchical difference: "The typical way of a journalist consulting us is that he stands behind my desk and looks over my shoulder, while I sit behind my computer and try to visualize what he has in mind." There is little room for the designer's expertise or ideas. The journalist does not only brief the designer on which story should be told, but also on how the story should be visualized. In the fast pace journalists need to work, there is hardly any time for elaborate briefings or discussions. To compensate for this the head of the graphics department started joining daily editorial meetings to anticipate possible news visualizations that journalists might want. While this form of boundary crossing could work in the long run, at the time of this research we perceived that the meetings were not aimed at the visualization of the news. We saw that there was only a one-sided boundary crossing in which the graphic department wanted to identify and understand the news processes, but the journalists were not willing enough the adjust the meetings to also discuss the visualization of the news.

The submissive role of the designer can be explained by the dominant broadcasting television culture in which journalists take a leading role and where designers take a quite modest position. According to the head of the graphics department designers tend to be more introvert than journalists: "While journalists tend to be very well-expressed, designers are more modest by nature. Therefore, designers find it hard to 'win' in conversations. As a designer you offer your own expertise, but that does not make you a journalist." Or as journalist clearly described, "They [the designers] are nice people, but their heart is not with making television. At times you cannot even seriously consult them as they have music playing while they are at work. They are just not real journalists. They don't consider it a sin to miss a deadline."

The case of the news organization shows that the fast-moving production process hinders discussions between the different experts to be able to understand each other and subsequently coordinate the work more effectively. Underlying 
this logistical obstruction of working pace we see that the dominant journalistic culture leaves little room for creating a shared mind-set on the objective of visualizations in the news. Over the past months, the management has again accentuated the importance of the use of visualizations, but without a shared mind-set an effective collaboration is difficult to reach.

\section{Interdisciplinary collaboration: Identification and coordination}

With this overview of the different forms of collaboration we can detect two major forms of trading zones: the one that encompasses the collaboration between an external client and a designer (external trading zone) and the trading zones within an organization between content producer and designer (internal trading zone).

When we look at the external trading zone, in the case of foster childcare the client imposed its ideas about the visualisation on the design agency, and the latter initially seemed to accept this rather submissive position. They did not apply their knowledge and expertise to help the client make the most suitable visualisation for the message they wanted to communicate. The deadlock in this trading zone was broken by the learning mechanism of identification, in which both parties realized that they had to take a step back in the process to identify which expertise is needed from whom. In the case of the recycling company the imposed trading zone worked out better as both the client and the design agency identified each other's expertise after which the client was able to take a more submissive role by leaving the design expertise to the agency. Once the expertise of both are identified, it is easier to coordinate the different tasks in the production process.

When we look at the internal trading zones we see a dominant role of the journalists or researchers towards the designers. This can be explained by the fact that the core business of the two organizations we studied, the broadcasting organization and the environmental agency, is not related to design or infographics, but to making content. At the animation agency that made an animation for the recycling company there was less of a submissive position of the designers as the core business of the animation agency is making visuals.

The submissive position of the designer or the hierarchal position of the socalled word people over the picture people shows that the learning mechanism of identification hardly took place. There is barely any room for understanding each other's roles or expertise, which makes it difficult to coordinate the different tasks in the whole production process. This problem of identification can also be explained by the fact that the different disciplines do not have a similar background and common language, let alone a common ground. This makes it difficult to identify with each other's expertise and possibilities. 
Broadly, then, this research has shown that there is not one collaboration form that can be proposed as the most ideal. While in some circumstances a fractionated trading zone works well, in other cases a dominated trading zone works more efficiently. Nevertheless, a common mind-set and objective is crucial to collaborate and create effective visualizations.

A solution to bringing these two groups together is to appoint a so-called transformer or a project manager who is responsible for managing the overall production process and bringing the different disciplines together. However, our research shows that merely appointing a transformer does not automatically mean an actual collaboration takes place or that the process goes more efficiently between the different disciplines. The involved disciplines and the transformer need to feel the need to collaborate and that is only felt when there is a common good the different disciplines are working for, or a boundary object that brings both parties closer to each other. Moreover, a transformer needs to accept a more mediating position in which he has affiliation with both the content as the design, while not leaning more towards a certain expertise or discipline.

\section{Discussion}

The field of infographics and visualisations is booming within media organizations and beyond. In this paper we studied the process of making information visualisations. In a growing interdisciplinary field we saw that collaboration between different disciplines does not always go efficiently, affecting the result of the visualization. Our research shows that a common ground between the involved experts is a precondition for creating the aimed objective. To analyse how this common ground develops as the discourse proceeds we used the concepts of trading zone and boundary crossing. That helped us to understand how the different forms of collaboration that we distinguished - enforced, dominated, fractionated, and attuned - might be effective in their specific circumstances. There are several factors that influence the interdisciplinary collaboration.

First and foremost, we found that there needs to be some understanding of and adaption to the iterative production process of information visualisation, as feedback loops are often indispensable due to the on-going interplay between verbal thinking and visual thinking.

Secondly, organizations with a dominant culture rooted in verbal thinking seem to be inclined to coercive forms of collaboration as far as the production of information visualisations is concerned. Our research shows that this led to underestimation of the skills and analytical competences of the designers. The designers were not involved in the problem-solving phase of the design process, with consequent sub-optimal designs. 
Thirdly, the size of the organisation influences the way people collaborate. In large corporations, having a separate design department - and as in our cases, one that is sometimes also psychically detached from the core business - makes it less accessible for the designers to collaborate with the other experts. At the same time, the fact that large organizations have a design department also shows a further professionalization of the field of design and infographics.

Another crucial factor is the type of client the infographic is meant for and which message the client wants to put across. A complex issue that needs to be communicated often demands an intensive phase of clearly articulating the message with the involved parties.

Lastly, the lead-time for making a visualisation is a crucial factor. While media organizations have to work with large time pressures, government organizations are accustomed to longer lead-times. This time also allows them to take more time to organize interdisciplinary meetings and to coordinate the interdisciplinary working process.

While many factors influence the production process of visualisations, our study shows how important it is to go back to basics in which the involved parties first have to be on the same page on which story has to be told before taking following steps. In order to get on the same page, it's important that different disciplines first identify each other's role and expertise. This step may be selfevident in theory, but our research has shown that it is not common in daily practice.

\section{References}

Akkerman, S. F., \& Bakker, A. (2011). Boundary crossing and boundary objects. Review of Educational Research, 81(2), 132-169.

Beak, S., Liebowitz, J., \& Lewis, M. (2000). An exploratory study: Supporting collaborative multimedia systems design. Multimedia Tools and Applications, $12,189-208$.

Collins, H. (2004). Interactional expertise as a third kind of knowledge. Phenomenology and Cognitive Sciences, 3, 125-143.

Collins, H., Evans, R., \& Gorman, M. (2007). Trading zones and interactional expertise. Studies in History and Philosophy of Science, 38, 657-666.

De Koning, B. B., Tabbers, H. K., Rikers, R. M. J. P., \& Paas, F. (2010). Attention guidance in learning from a complex animation: Seeing is understanding? Learning and Instruction, 20(2), 111-122.

Galison, P. (1997). Image and logic: A material culture of microphysics. Chicago: University of Chicago Press.

Giardina, M., \& Medina, P. (2013). Information graphics design challenges and w orkflow management. Online Journal of Communication and Media Technologies, 3(1), 108-124. 
Heiser, J., \& Tversky, B. (2002). Diagrams and descriptions in acquiring complex systems. Proceedings of the meetings of the Cognitive Society. Erlbaum: Hillsdale, N.J.

Kirk, A. (2012). Data visualisation: A successful design process. BirminghamMumbai: Packt Publishing.

Kleinsman, M., Valkenburg., \& Buijs, J. (2007). Why do(n't) actors in collaborative design understand each other? An empirical study towards a better understanding of collaborative design. CoDesign, 3(1), 59-73.

Lankow, J., Ritchie, J., \& Crooks, R. (2012). Infographics: the power of visual storytelling. New Jersey: John Wiley \& Sons.

Mora, G. (2012, November 7). Journalists and infographic-designers: a love-hate relationship (part one). [Web log post]. Retrieved via http://visualoop.com/3134/journalists-and-infographic-designers-a-love-haterelationship-part-one

Segel, E. and Heer, J. (2010). Narrative Visualization: Telling Stories with Data. Journal IEEE Transactions on Visualization and Computer Graphics 16 (6), 1139-1148.

Smit, G., de Haan, Y., \& Buijs, L. (2014). Visualising news: make it work. Digital Journalism. http://dx.doi.org/10.1080/21670811.2014.897847.

Star, S. L., \& Griesemer, J. R. (1989). Institutional ecology, translations and boundary objects: Amateurs and professionals in Berkeley's Museum of Vertebrate Zoology. Social Studies of Science. 19, 387-420.

Weber, W. \& Rall, H. (2012). Data visualisation in online journalism and its implications for the production process. Paper presented at the $16^{\text {th }}$ International Conference on Information Visualisation, The University of Montpellier, Montpellier, July 11-13, 2012.

http://dx.doi.org/10.1109/IV.2012.65 